\title{
Extended warm and dense gas towards W49A: starburst conditions in our Galaxy?
}

\author{
Z. Nagy ${ }^{1,2}$, F. F. S. van der Tak ${ }^{2,1}$, G. A. Fuller ${ }^{3}$, M. Spaans ${ }^{1}$, and R. Plume ${ }^{4}$ \\ 1 Kapteyn Astronomical Institute, University of Groningen, PO Box 800, 9700 AV Groningen, The Netherlands \\ e-mail: nagy@astro.rug.nl \\ 2 SRON Netherlands Institute for Space Research, PO Box 800, 9700 AV Groningen, The Netherlands \\ 3 Jodrell Bank Centre for Astrophysics, School of Physics and Astronomy, University of Manchester, Manchester, M13 9PL, UK \\ ${ }^{4}$ Department of Physics and Astronomy, University of Calgary, Calgary, T2N 1N4, AB, Canada
}

Received 5 December 2011 / Accepted 28 March 2000

\section{ABSTRACT}

Context. The star formation rates in starburst galaxies are orders of magnitude higher than in local star-forming regions, and the origin of this difference is not well understood.

Aims. We use sub-mm spectral line maps to characterize the physical conditions of the molecular gas in the luminous Galactic starforming region W49A and compare them with the conditions in starburst galaxies.

Methods. We probe the temperature and density structure of W49A using $\mathrm{H}_{2} \mathrm{CO}$ and $\mathrm{HCN}$ line ratios over a $2^{\prime} \times 2^{\prime}(6.6 \times 6.6 \mathrm{pc})$ field with an angular resolution of $15^{\prime \prime}(\sim 0.8 \mathrm{pc})$ provided by the JCMT Spectral Legacy Survey. We analyze the rotation diagrams of lines with multiple transitions with corrections for optical depth and beam dilution, and estimate excitation temperatures and column densities.

Results. Comparing the observed line intensity ratios with non-LTE radiative transfer models, our results reveal an extended region (about $1^{\prime} \times 1^{\prime}$, equivalent to $\sim 3 \times 3 \mathrm{pc}$ at the distance of W49A) of warm $(>100 \mathrm{~K})$ and dense $\left(>10^{5} \mathrm{~cm}^{-3}\right)$ molecular gas, with a mass of $2 \times 10^{4}-2 \times 10^{5} M_{\odot}$ (by applying abundances derived for other regions of massive star-formation). These temperatures and densities in W49A are comparable to those found in clouds near the center of the Milky Way and in starburst galaxies.

Conclusions. The highly excited gas is likely to be heated via shocks from the stellar winds of embedded, O-type stars or alternatively due to UV irradiation, or possibly a combination of these two processes. Cosmic rays, X-ray irradiation and gas-grain collisional heating are less likely to be the source of the heating in the case of W49A.

Key words. stars: formation - ISM: molecules - ISM: individual objects: W49A

\section{Introduction}

The formation of stars proceeds on scales ranging from 1000 AU-sized isolated low-mass cores to $\sim 10$ pc-sized giant molecular clouds with embedded massive clusters. The observed levels of star-formation activity also vary greatly: from $\sim 10^{-4} M_{\odot} /$ yr in dwarf galaxies like I Zw 18 (Aloisi et al. 1999) to $\sim 3 M_{\odot} /$ yr in the Milky Way to $\sim 10^{3} M_{\odot} /$ yr in starburst galaxies (e.g. Solomon \& Vanden Bout 2005). The origin of this large spread in star formation rate may lie in different physical conditions with the gas forming the stars, the nature and role of feedback effects or other mechanisms. As one of the the most active star-forming regions in the Galactic disk, W49A provides a relatively nearby laboratory in which to study intense star formation activity and may provide insights into explaining the large observed range in star-formation activity in galaxies.

W49A is a luminous $\left(>10^{7} L_{\odot}\right)$ and massive $\left(\sim 10^{6} M_{\odot}\right)$ starforming region (Sievers et al. 1991) at a distance of $11.4 \mathrm{kpc}$ (Gwinn et al. 1992). W49A contains several signatures of high star-formation activity, in particular, strong $\mathrm{H}_{2} \mathrm{O}$ maser emission (Genzel et al. 1978). Line ratios of $\mathrm{HNC}, \mathrm{HCN}$ and $\mathrm{HCO}^{+}$and their isotopologues suggest similar conditions to starburst galaxies (Roberts et al. 2011). Several ideas have been proposed to explain the high star-formation activity of W49A.

Welch et al. (1987) propose a large-scale inside-out gravitational collapse by interpreting two velocity components of $\mathrm{HCO}^{+} J=1-0$ spectra measured toward the ring-like configuration of UC HII regions in terms of an infall profile. Serabyn et al. (1993) interpret the two-components of the double-peak line profile seen in CS $(J=10-9,7-6,5-4$, $3-2)$ and $C^{34} S(J=10-9,7-6,5-4)$ transitions as coming from different clouds, and suggest that massive star formation in W49A is triggered by a large-scale cloud merger. Williams et al. (2004) argue that one cannot distinguish between a global collapse model of a large cloud and a multiple-cloud model, based on $\mathrm{HCO}^{+}(J=3-2$ and $1-0)$ and $\mathrm{C}^{18} \mathrm{O}(J=2-1)$ data. Roberts et al. (2011) observe single-peaked $\mathrm{HC}^{18} \mathrm{O}^{+}(J=4-3)$ line profiles, which supports the large-scale infall hypothesis. In ${ }^{13} \mathrm{CO}$ and $\mathrm{C}^{18} \mathrm{O} J=2-1$ maps of the W49A region Peng et al. (2010) find evidence of expanding shells with the dense clumps in the region lying peripherally along the shells. These expanding shells could provide a natural explanation of the observed molecular line profiles, and triggered massive star formation in W49A.

This paper reports new kinetic temperature and density estimates for a $2^{\prime} \times 2^{\prime}$ region of $\mathrm{W} 49 \mathrm{~A}$ based on single dish, JCMT images with $15^{\prime \prime}$ resolution. These indicate the presence of extended warm and dense gas towards the central region of the source. We use these results to compare W49A to starburst galaxies where similar results have been reported.

\section{Observations and data reduction}

This paper presents results from data obtained as part of the JCMT Spectral Legacy Survey (SLS, Plume et al. 2007) 


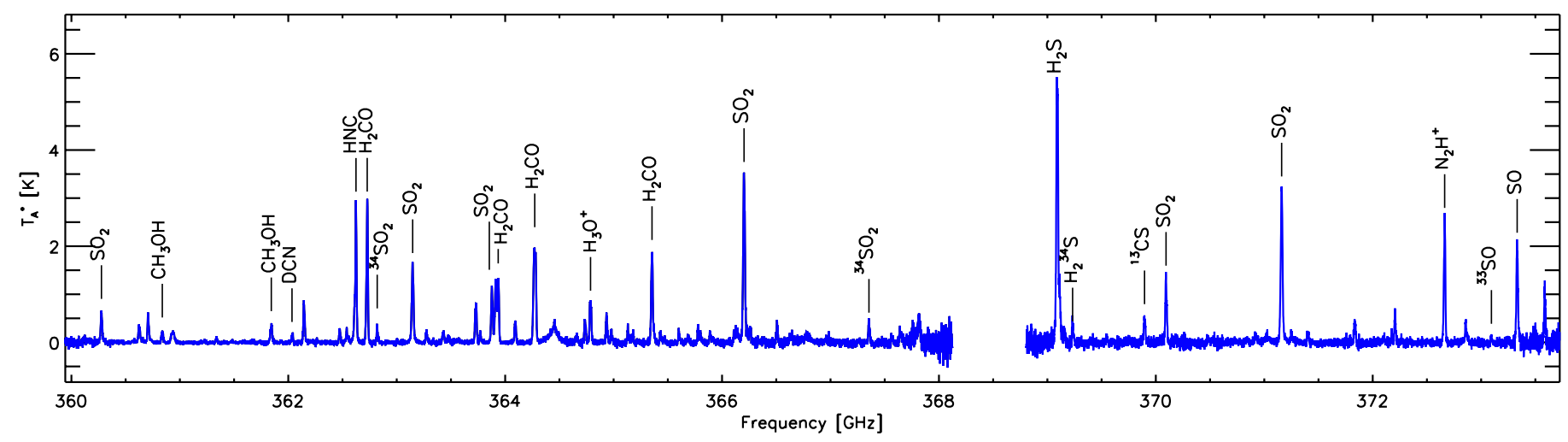

Fig. 1. The spectrum towards the source center $(\mathrm{RA}(\mathrm{J} 2000)=19: 10: 13.4 ; \operatorname{Dec}(\mathrm{J} 2000)=09: 06: 14)$ in the 360-373 GHz frequency range of the SLS. The main detected lines are labeled. The gap in the spectrum around $368.5 \mathrm{GHz}$ is a region of high atmospheric extinction and was not observed.

covering between 330 and $373 \mathrm{GHz}$ which has been carried out at the James Clerk Maxwell Telescope $\left(\mathrm{JCMT}^{1}\right.$ on Mauna Kea, Hawai'i. The data were taken using the HARP-B receiver and the ACSIS correlator (Buckle et al. 2009). HARP-B consists of 16 pixels or receptors separated by 30 arcsec with a foot print of 2 arcmin.

The observations were carried out using jiggle-position switch mode. Each map covers a $2^{\prime} \times 2^{\prime}$ field sampled every $7.5^{\prime \prime}$, half of the JCMT beam width at the relevant frequencies $\left(15^{\prime \prime}\right.$ at $\left.345 \mathrm{GHz}\right)$. The spectral resolution at these frequencies is $\sim 0.8 \mathrm{~km} \mathrm{~s}^{-1}$ and the beam efficiency is 0.63 (Buckle et al. 2009). The spectra were taken using an off-position $14^{\prime}$ to the northeast of the source.

Data reduction was done manually using the tools in the Starlink software package. The raw time series files are processed in $1 \mathrm{GHz}$ wide chunks. These files were inspected for bad receptors, baselines and frequency spikes. Bad receptors, bad baselines and spikes were masked in the time series files before they have been converted to three-dimensional data cubes $(\mathrm{RA} \times$ Dec $\times$ frequency). These $3 \mathrm{D}$ cubes then were baseline corrected by fitting and removing a linear or second-order polynomial baseline. The baseline corrected cubes are then mosaicked to a larger cube covering an area of $2^{\prime} \times 2^{\prime}$ and the frequency range of interest. Single sideband system temperatures for these observations are in the range between $255 \mathrm{~K}$ (at $360 \mathrm{GHz}$ ) and $700 \mathrm{~K}$ (at $373 \mathrm{GHz}$ ). The analysis here primarily focuses on a number of spectral lines in the $360-373 \mathrm{GHz}$ range plus a few selected lines from the $330-360 \mathrm{GHz}$ part of the survey. The whole, systematic data analysis of the full SLS frequency range is the focus of a future paper.

We also use ancillary observations of the $\mathrm{HCN} J=3-2$ transition made using JCMT raster maps with half-beamwidth spacing using the receiver A $(211-276 \mathrm{GHz}$; beamwidth 20"; main beam efficiency: 0.69). The HCN 3-2 map covers approximately the same field that of the SLS HARP maps. The HCN 3-2 data were reduced and calibrated with standard Starlink procedures. Linear baselines were removed and the data were Hanning smoothed. The map is a grid of 15 pixel $\times 15$ pixels, where each pixel covers $8^{\prime \prime} \times 8^{\prime \prime}$.

\footnotetext{
1 The James Clerk Maxwell Telescope is operated by the Joint Astronomy Centre on behalf of the Science and Technology Facilities Council of the United Kingdom, The Netherlands Organisation for Scientific Research, and the National Research Council of Canada.
}

Table 1. Summary of the molecular lines used in this paper.

\begin{tabular}{|c|c|c|c|c|}
\hline Molecule & Transition & $\begin{array}{c}\text { Frequency } \\
(\mathrm{MHz})\end{array}$ & $\begin{array}{l}E_{\text {up }} \\
(\mathrm{K}) \\
\end{array}$ & $\begin{array}{c}\operatorname{rms}\left(T_{\mathrm{mb}}\right)^{a} \\
(\mathrm{~K})\end{array}$ \\
\hline $\mathrm{HCN}$ & $J=3-2$ & 265886.2 & 25.5 & 0.90 \\
\hline $\mathrm{HCN}$ & $J=4-3$ & 354505.5 & 42.5 & 0.05 \\
\hline $\mathrm{H}_{2} \mathrm{CO}$ & $5_{1,5}-4_{1,4}$ & 351768.6 & 62.5 & 0.12 \\
\hline $\mathrm{H}_{2} \mathrm{CO}$ & $5_{0,5}-4_{0,4}$ & 362736.1 & 52.3 & 0.05 \\
\hline $\mathrm{H}_{2} \mathrm{CO}$ & $5_{2,4}-4_{2,3}$ & 363945.9 & 99.5 & 0.06 \\
\hline $\mathrm{H}_{2} \mathrm{CO}$ & $5_{4,1}-4_{4,0}$ & 364103.3 & 240.7 & 0.06 \\
\hline $\mathrm{H}_{2} \mathrm{CO}$ & $5_{3,3}-4_{3,2}$ & 364275.1 & 158.4 & 0.08 \\
\hline $\mathrm{H}_{2} \mathrm{CO}$ & $5_{3,2}-4_{3,1}$ & 364288.9 & 158.4 & 0.08 \\
\hline $\mathrm{H}_{2} \mathrm{CO}$ & $5_{2,3}-4_{2,2}$ & 365363.4 & 99.7 & 0.06 \\
\hline $\mathrm{CH}_{3} \mathrm{OH}$ & $11_{0,11}-10_{1,9}$ & 360848.9 & 166.0 & 0.04 \\
\hline $\mathrm{CH}_{3} \mathrm{OH}$ & $8_{1,7}-7_{2,5}$ & 361852.3 & 104.6 & 0.05 \\
\hline $\mathrm{CH}_{3} \mathrm{OH}$ & $16_{1,15}-16_{0,16}$ & 363440.3 & 332.6 & 0.06 \\
\hline $\mathrm{CH}_{3} \mathrm{OH}$ & $7_{2,5}-6_{1,5}$ & 363739.8 & 87.3 & 0.06 \\
\hline $\mathrm{CH}_{3} \mathrm{OH}$ & $17_{1,16}-17_{0,17}$ & 371415.5 & 372.4 & 0.09 \\
\hline $\mathrm{SO}_{2}$ & $34_{5,29}-34_{4,30}$ & 360290.4 & 611.9 & 0.06 \\
\hline $\mathrm{SO}_{2}$ & $20_{8,12}-21_{7,15}$ & 360721.8 & 349.8 & 0.04 \\
\hline $\mathrm{SO}_{2}$ & $21_{4,18}-21_{3,19}$ & 363159.3 & 252.1 & 0.05 \\
\hline $\mathrm{SO}_{2}$ & $24_{1,23}-24_{0,24}$ & 363890.9 & 280.5 & 0.06 \\
\hline $\mathrm{SO}_{2}$ & $23_{2,22}-23_{1,23}$ & 363925.8 & 259.9 & 0.06 \\
\hline $\mathrm{SO}_{2}$ & $25_{9,17}-26_{8,18}$ & 364950.1 & 497.1 & 0.06 \\
\hline $\mathrm{SO}_{2}$ & $15_{2,14}-14_{1,13}$ & 366214.5 & 119.3 & 0.09 \\
\hline $\mathrm{SO}_{2}$ & $9_{6,4}-10_{5,5}$ & 370108.6 & 129.7 & 0.11 \\
\hline $\mathrm{SO}_{2}$ & $6_{3,3}-5_{2,4}$ & 371172.5 & 41.4 & 0.09 \\
\hline
\end{tabular}

Notes. ${ }^{(a)}$ Noise level measured over the central $1^{\prime} \times 1.5^{\prime}$, for $1 \mathrm{~km} \mathrm{~s}^{-1}$ channels.

\section{Results}

\subsection{Line selection}

Figure 1 shows the $360-373 \mathrm{GHz}$ spectrum towards the central position $(\operatorname{RA}(J 2000)=19: 10: 13.4 ; \operatorname{Dec}(J 2000)=09: 06: 14)$. More than 80 lines have been identified in this spectrum.

Our initial selection of lines for the analysis here is based on molecules that show multiple transitions in the high-frequency range (360-373 GHz) of the SLS, which include six $\mathrm{H}_{2} \mathrm{CO}$, five $\mathrm{CH}_{3} \mathrm{OH}$ and nine $\mathrm{SO}_{2}$ transitions (Table 1). These are used to trace the excitation conditions. One more $\mathrm{H}_{2} \mathrm{CO}$ transition from the lower frequency part of the survey $\left(\mathrm{H}_{2} \mathrm{CO} 5_{15}-4_{14}\right)$ was added to be used as a tracer of kinetic temperatures, together with the $5_{33}-4_{32}$ transition from the high-frequency part of the 
SLS. The HCN 4-3 transition was selected from the lower frequency range, to trace the density structure, together with the HCN 3-2 transition.

In this paper, we restrict our analysis to our line selection summarized above and in Table 1. A subsequent paper (Nagy et al., in prep.) will give more details on the chemical inventory including the full SLS frequency range $(330-373 \mathrm{GHz})$ and on kinematics traced by various species with significant spatial extension. Figure 2 shows the main regions around the center of W49A overplotted on the integrated intensity maps in the $\mathrm{HCN} 4-3, \mathrm{SO}_{2} 15_{2,14}-14_{1,13}, \mathrm{CH}_{3} \mathrm{OH} 7_{2,5}-6_{1,5}$ and $\mathrm{H}_{2} \mathrm{CO} 5_{15}-4_{14}$ lines. The integrated intensities of the HCN and $\mathrm{H}_{2} \mathrm{CO}$ lines show a similar distribution, indicating that their emission originates in the same volume. This is also supported by the line profiles of these two species (Nagy et al., in prep.). In the following sections, we will refer to these regions with the coordinates indicated in the figure: source center $(\mathrm{RA}(\mathrm{J} 2000)=19: 10: 13.4 ; \operatorname{Dec}(\mathrm{J} 2000)=09: 06: 14)$, eastern tail $(\mathrm{RA}(\mathrm{J} 2000)=19: 10: 16.6 ; \operatorname{Dec}(\mathrm{J} 2000)=09: 05: 48)$, northern clump $(\mathrm{RA}(\mathrm{J} 2000)=19: 10: 13.6 ; \operatorname{Dec}(\mathrm{J} 2000)=09: 06: 48)$ and southwest clump $(\operatorname{RA}(J 2000)=19: 10: 10.6 ; \operatorname{Dec}(J 2000)=$ 09:05:18).

\subsection{Excitation conditions}

A well known method for the estimation of excitation temperatures and column densities is the rotational diagram method (e.g. Turner 1991). It can be applied to molecules with multiple observed transitions using three assumptions: 1 ) the lines are optically thin, 2) the level populations can be characterized by a single excitation temperature ("rotational temperature", $T_{\text {rot }}$ ) and 3) the emission is homogeneous and fills the telescope beam. Then the measured integrated main-beam temperatures of lines ( $\int T_{\mathrm{MB}} \mathrm{d} V \mathrm{~K} \mathrm{~km} \mathrm{~s}^{-1}$ ) is related to the column densities of the molecules in the upper level $\left(N_{\mathrm{u}}\right)$ by:

$\frac{N_{\mathrm{u}}}{g_{\mathrm{u}}}=\frac{N_{\mathrm{tot}}}{Q\left(T_{\text {rot }}\right)} \exp \left(-\frac{E_{\mathrm{u}}}{T_{\text {rot }}}\right)=\frac{1.67 \times 10^{14}}{v \mu^{2} S} \int T_{\mathrm{MB}} \mathrm{d} V$,

with $g_{\mathrm{u}}$ the statistical weight of level u, $N_{\text {tot }}$ the total column density in $\mathrm{cm}^{-2}, Q\left(T_{\text {rot }}\right)$ the partition function for $T_{\text {rot }}, E_{\mathrm{u}}$ the upper level energy in $\mathrm{K}, v$ the frequency in $\mathrm{GHz}, \mu$ the permanent dipole moment in Debye and $S$ the line strength value. A linear fit to $\ln \left(N_{\mathrm{u}} / g_{\mathrm{u}}\right)-E_{\mathrm{u}}$ gives $T_{\text {rot }}$ as the inverse of the slope, and $N_{\text {tot }}$, the column density can be derived. The rotational temperature would be expected to be equal to the kinetic temperatures if all levels were thermalized.

In the case of non-LTE excitation, a different excitation temperature may characterize the population of each level relative to that of the ground state or relative to that of any other level. In this case, the three assumptions mentioned for the rotation diagram method do not apply. Equation (1) can be modified to include the effect of optical depth $\tau$ through the factor $C_{\tau}=\tau /\left(1-\mathrm{e}^{-\tau}\right)$ and beam dilution $f\left(=\Omega_{s} / \Omega_{a}\right)$ (Goldsmith \& Langer 1999), with $\Omega_{s}$ the size of the emission region and $\Omega_{a}$ the size of the telescope beam.

$\ln \left(\frac{N_{\mathrm{u}}}{g_{\mathrm{u}}}\right)=\ln \left(\frac{N_{\mathrm{tot}, 1}}{Q\left(T_{\mathrm{rot}}\right)}\right)-\frac{E_{\mathrm{u}}}{k T_{\mathrm{ex}}}+\ln (f)-\ln \left(C_{\tau}\right)$.

According to Eq. (2), for a given upper level, $N_{\mathrm{u}}$ can be evaluated from a set of $N_{\mathrm{tot}, 1}, T_{\mathrm{ex}}, f$ and $C_{\tau}$. Since $C_{\tau}$ is a function of $N_{\text {tot, } 1}$ and $T_{\text {ex }}$, the independent parameters are therefore $N_{\text {tot, } 1}$, $T_{\text {ex }}$ and $f$. Solving the equation for a "source size" between 1 "

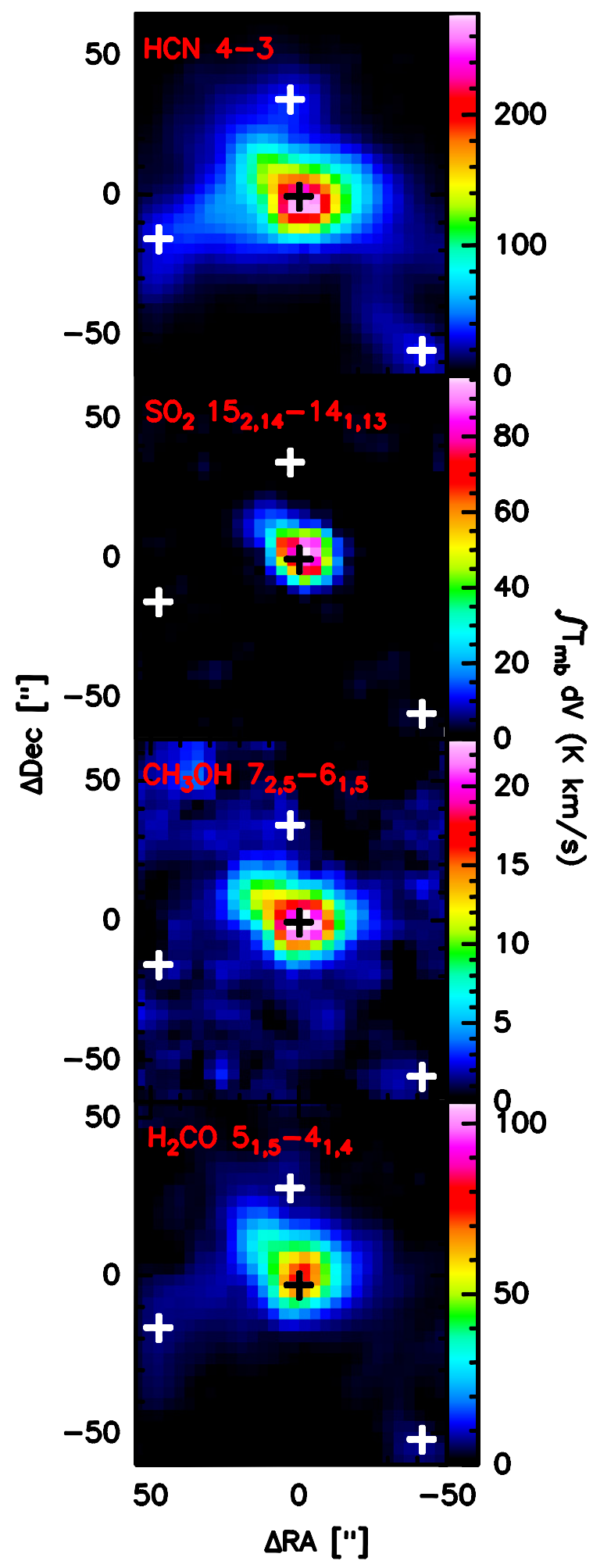

Fig. 2. The integrated intensity distribution of $\mathrm{HCN} 4-3, \mathrm{SO}_{2}$ $15_{2,14}-14_{1,13}, \mathrm{CH}_{3} \mathrm{OH} 7_{2,5}-6_{1,5}$ and $\mathrm{H}_{2} \mathrm{CO} 5_{15}-4_{14}$. The central position is shown with black plus signs, the off-center regions (northern clump, eastern tail and southwest clump) are also shown with white plus signs.

and $15^{\prime \prime}$ (which is equivalent with a beam dilution factor between $(1 / 15)^{2}$ and uniform beam filling), $\chi^{2}$ minimization gives $N_{\text {tot, } 1}$ and $T_{\text {ex }}$. Figure 3 shows the rotation diagrams for $\mathrm{CH}_{3} \mathrm{OH}$, $\mathrm{H}_{2} \mathrm{CO} \& \mathrm{SO}_{2}$ toward the central position with (green symbols) and without (red symbols) taking into account the optical depth 


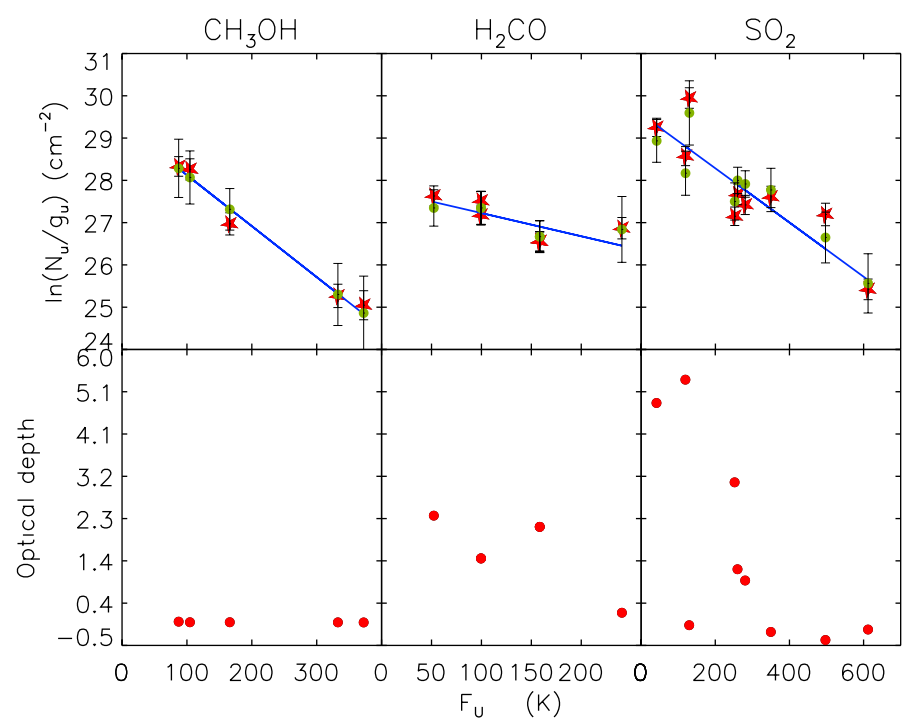

Fig. 3. The results of the rotation diagram analysis for $\mathrm{H}_{2} \mathrm{CO}$ toward the center. Top panels: the results of the rotation diagram analysis before (red symbols) and after (green symbols) corrections for optical depth and beam dilution. The overplotted blue line corresponds to a linear fit to the rotational diagram without corrections. Bottom panels: the corresponding best fit optical depths from the $\chi^{2}$ minimization.

Table 2. Results of the excitation analysis toward the center of W49A.

\begin{tabular}{l|ccc}
\hline \hline & \multicolumn{3}{|c}{ I. Rotation diagrams } \\
\hline & $\mathrm{SO}_{2}$ & $\mathrm{CH}_{3} \mathrm{OH}$ & $\mathrm{H}_{2} \mathrm{CO}$ \\
\hline$T_{\text {rot }}[\mathrm{K}]$ & $156 \pm 11$ & $83 \pm 7$ & $182 \pm 84$ \\
$N_{\text {tot }}{ }^{a}\left[10^{15} \mathrm{~cm}^{-2}\right]$ & $14.56 \pm 2.99$ & $6.58 \pm 1.38$ & $0.78 \pm 0.17$ \\
Source size $\left[{ }^{\prime \prime}\right]$ & $3.1_{-0.7}^{+0.0}$ & $12.5_{-10.7}^{+2.5}$ & $2.4_{-0.3}^{+4.2}$ \\
$T_{\text {ex }}[\mathrm{K}]$ & $115_{-15}^{+40}$ & $82.1_{-16.1}^{+16.1}$ & $138.2_{-115.0}^{+111.3}$ \\
$N_{\text {tot }, 1}{ }^{b}\left[10^{15} \mathrm{~cm}^{-2}\right]$ & $1400.0_{-430.0}^{+400.0}$ & $9.5_{-3.2}^{+86.5}$ & $43_{-25}^{+1857}$ \\
\hline & \multicolumn{3}{|c}{$\mathrm{II}$ Non-LTE calculation } \\
\hline$T_{\text {kin }}[\mathrm{K}]^{c}$ & \multicolumn{3}{|c}{300} \\
$N\left[\mathrm{H}_{2} \mathrm{CO}\right]\left[\mathrm{cm}^{-2}\right]$ & $3.5 \times 10^{14}$ \\
$N[\mathrm{HCN}]\left[\mathrm{cm}^{-2}\right]$ & \multicolumn{3}{|c}{$7.3 \times 10^{15}$} \\
$n\left[\mathrm{~cm}^{-3}\right]^{d}$ & \multicolumn{3}{|c}{$1.8 \times 10^{6}$} \\
\hline
\end{tabular}

Notes. ${ }^{(a)}$ Total beam-averaged column density calculated using the assumption that the lines are optically thin, the level populations can be characterized by a single excitation temperature and the emission fills the telescope beam. ${ }^{(b)}$ Total source-averaged column density given by the $\chi^{2}$ minimization, including a correction for the optical depth and a beam dilution factor. The errors show the $1-\sigma$ upper and lower limits of the best fit. ${ }^{(c)}$ The uncertainty of the kinetic temperatures is $\sim 30 \%$. (d) The uncertainty of the density estimates is a factor of 2.

and beam dilution. Table 2 includes the parameters of the rotation diagram fit toward the central position.

The $\mathrm{CH}_{3} \mathrm{OH}$ lines are found to be optically thin $(\tau=$ $0.004-0.02$ ), so the rotation diagram fit is a good approximation to the column densities and excitation temperatures. The derived rotation temperature is $83 \pm 7 \mathrm{~K}$. The $\mathrm{H}_{2} \mathrm{CO}$ and $\mathrm{SO}_{2}$ lines are mostly optically thick with optical depths in the range of 0.2-2.35 for $\mathrm{H}_{2} \mathrm{CO}$ and $0.1-5.4$ for $\mathrm{SO}_{2}$. The best fit excitation temperatures are $138 \mathrm{~K}$ for $\mathrm{H}_{2} \mathrm{CO}$ and $115 \mathrm{~K}$ for $\mathrm{SO}_{2}$. The best fit total column densities are $4.3 \times 10^{16} \mathrm{~cm}^{-2}$ for $\mathrm{H}_{2} \mathrm{CO}$ and $1.4 \times 10^{18} \mathrm{~cm}^{-2}$ for $\mathrm{SO}_{2}$. The $\chi^{2}$ minimization for $\mathrm{H}_{2} \mathrm{CO}$ and $\mathrm{SO}_{2}$ results in a best fit "source size" of $\sim 2-3$ ", which is equivalent with $0.11-0.17 \mathrm{pc}$ for W49A. This is consistent with the

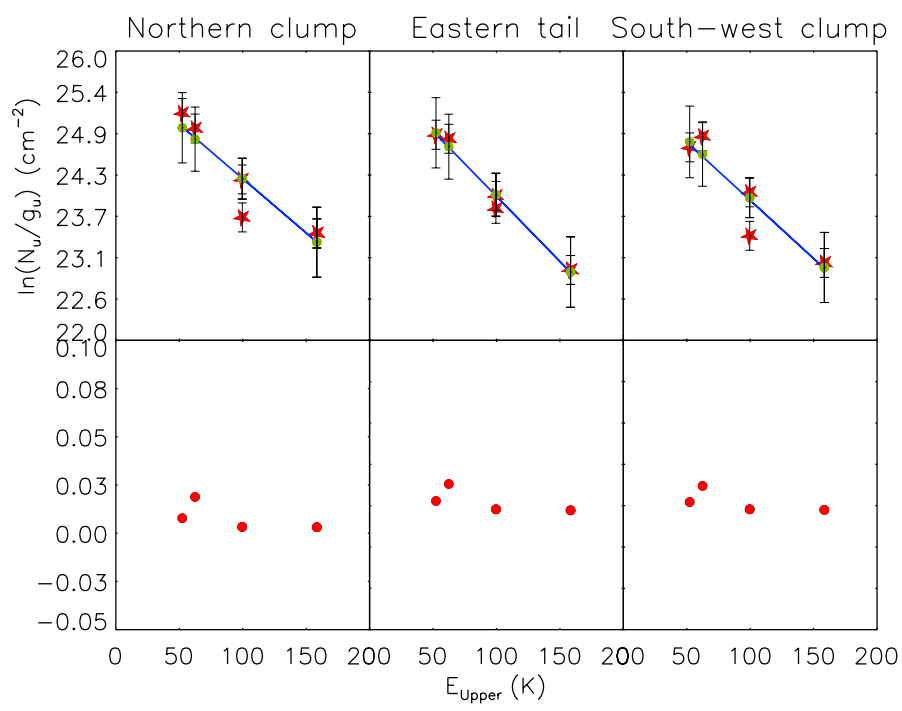

Fig. 4. The results of the rotation diagram analysis for $\mathrm{H}_{2} \mathrm{CO}$ at offcenter positions. Top panels: the results of the rotation diagram analysis before (red symbols) and after (green symbols) corrections for optical depth and beam dilution. The overplotted blue line corresponds to a linear fit to the rotational diagram without corrections. Bottom panels: the corresponding best fit optical depths from the $\chi^{2}$ minimization.

Table 3. Results of the excitation analysis toward the off-center regions of W49A.

\begin{tabular}{l|ccc}
\hline \hline & \multicolumn{3}{|c}{ I. Rotation diagrams } \\
\hline & Northern clump & Eastern tail & Southwest clump \\
\hline$T_{\text {rot }}[\mathrm{K}]$ & $67 \pm 13$ & $56 \pm 3$ & $62 \pm 11$ \\
$N_{\text {tot }}{ }^{a}\left[10^{13} \mathrm{~cm}^{-2}\right]$ & $2.26 \pm 0.48$ & $1.82 \pm 0.46$ & $1.68 \pm 0.36$ \\
Source size $\left[{ }^{\prime \prime}\right]$ & $14.44_{-11.2}^{+0.56}$ & $14.44_{-11.2}^{+0.56}$ & $14.44_{-10.64}^{+0.56}$ \\
$T_{\text {ex }}[\mathrm{K}]$ & $67_{-13}^{+21}$ & $55_{-9}^{+14}$ & $61_{-10}^{+19}$ \\
$N_{\text {tot, }, 1}{ }^{b}\left[10^{13} \mathrm{~cm}^{-2}\right]$ & $2.2_{-0.2}^{+44.8}$ & $1.9_{-0.3}^{+39.1}$ & $1.7_{-0.2}^{+22.3}$ \\
\hline & \multicolumn{3}{|c}{ II. Non-LTE calculation } \\
\hline & Northern clump & Eastern tail & Southwest clump \\
\hline$T_{\text {kin }}[\mathrm{K}]^{c}$ & \multicolumn{4}{c}{68} \\
$N\left[\mathrm{H}_{2} \mathrm{CO}\right]\left[\mathrm{cm}^{-2}\right]$ & $0.7-1.1 \times 10^{14}$ & $4.9-5.0 \times 10^{13}$ & $3.2-4.5 \times 10^{14}$ \\
$N[\mathrm{HCN}]\left[\mathrm{cm}^{-2}\right]$ & $1.5-2 \times 10^{14}$ & $7-8 \times 10^{13}$ & $6.2-9.6 \times 10^{14}$ \\
$n\left[\mathrm{~cm}^{-3}\right]^{d}$ & $5.6 \times 10^{5}$ & $1.2 \times 10^{6}$ & $1.3 \times 10^{5}$ \\
\hline
\end{tabular}

Notes. ${ }^{(a)}$ Total beam-averaged column density calculated using the assumption that the lines are optically thin, the level populations can be characterized by a single excitation temperature and the emission fills the telescope beam. ${ }^{\left({ }^{b}\right)}$ Total source-averaged column density given by the $\chi^{2}$ minimization, including a correction for the optical depth and a beam dilution factor. The errors show the $1-\sigma$ upper and lower limits of the best fit. ${ }^{(c)}$ The uncertainty of the kinetic temperatures is $\sim 30 \%$. (d) The uncertainty of the density estimates is a factor of 2 .

sizes of hot cores and UC HII regions resolved by sub-arcsecond resolution measurements, such as Wilner et al. (2001), De Pree et al. (1997, 2004).

Figure 4 shows the rotation diagrams for $\mathrm{H}_{2} \mathrm{CO}$ toward the three main regions outside the center. The rotation diagram with corrections for optical depth and beam dilution (Fig. 4 and Table 3) gives similar excitation conditions toward the three main sub-regions (excitation temperatures: $67 \mathrm{~K}$ for the northern clump, $56 \mathrm{~K}$ for the eastern tail and $61 \mathrm{~K}$ for the southwest 

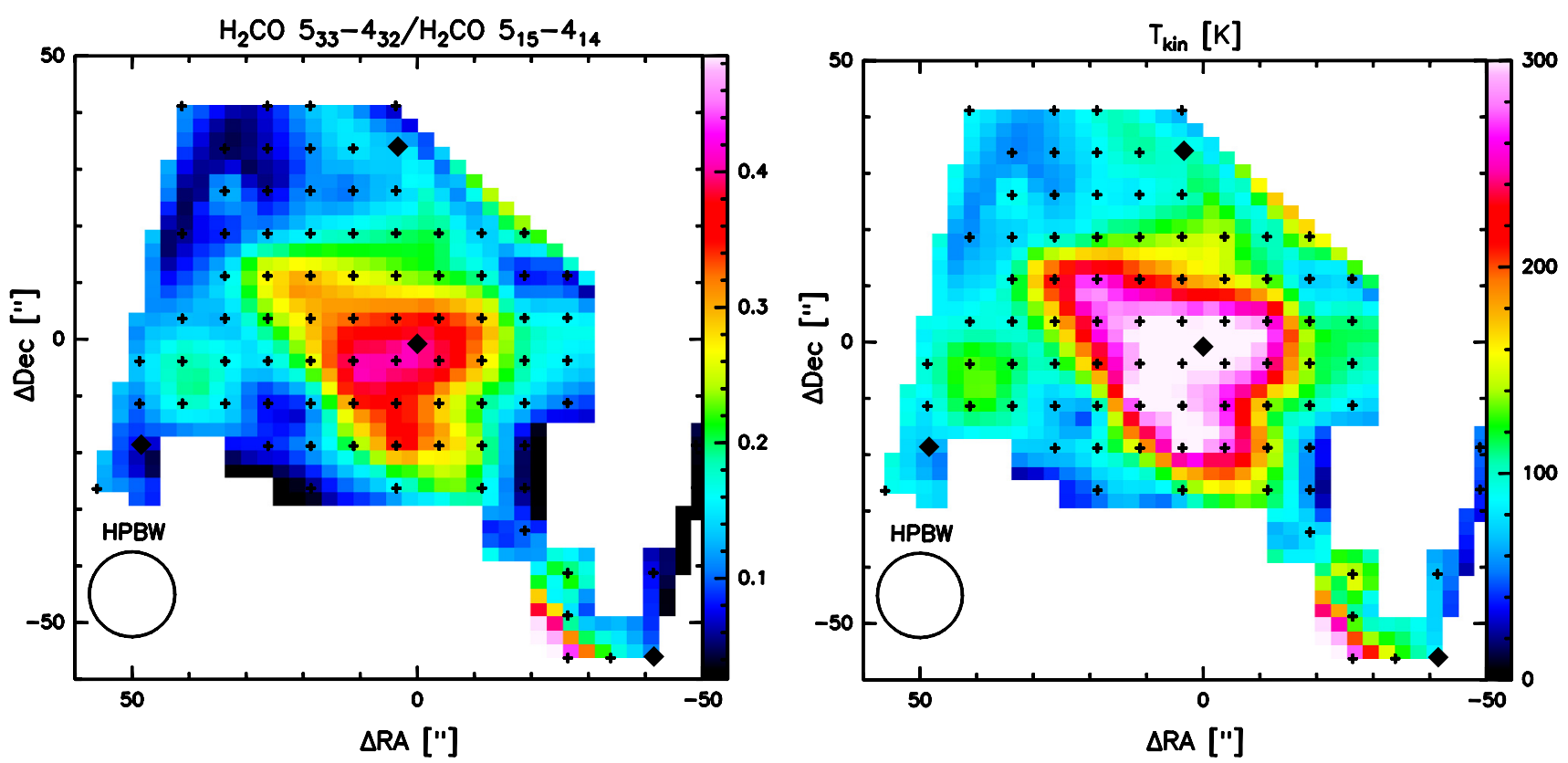

Fig. 5. Left panel: ratio of $5_{33}-4_{32}$ to the $5_{15}-4_{14} \mathrm{H}_{2} \mathrm{CO}$ transition. Right panel: kinetic temperature map of W49A determined from line ratio shown in the left panel.

clump). These excitation temperatures are about half of the excitation temperature towards the central position (138 K). Since the lines are optically thin, the rotation temperatures are good estimates for the excitation temperatures. The best fit column densities are also similar between the off-center positions, about $1.7-2.2 \times 10^{13} \mathrm{~cm}^{-3}$, almost three orders of magnitude less than the best fit column density towards the center. The data at these positions are consistent with optically thin emission and uniform beam filling.

\subsection{Kinetic temperature estimates}

To estimate the local physical conditions in W49A, we use ratios of lines from the same molecular species. The kinetic temperature can be probed by the line ratios of $\mathrm{H}_{2} \mathrm{CO}$ lines from the same $J$-state but different $K$-states. In this case, we use the $\mathrm{o}-\mathrm{H}_{2} \mathrm{CO}$ $5_{15}-4_{14}(351.769 \mathrm{GHz})$ and $\mathrm{o}-\mathrm{H}_{2} \mathrm{CO} 5_{33}-4_{32}(364.275 \mathrm{GHz})$ transitions, since they have been detected in a large part of the SLS field and have been found to be excellent tracers of kinetic temperature (Fig. A.1). Our calculations use the non-LTE radiative transfer program Radex (Van der Tak et al. 2007), molecular data file from the LAMDA database (Schöier et al. 2005) and $\mathrm{H}_{2} \mathrm{CO}$ collision data from Green (1991), which have been scaled by 1.37 to make a first order approximation for collisions with $\mathrm{H}_{2}$. Uncertainties introduced by using these rates may be up to $50 \%$ for the column densities, but less affect the densities and temperatures, since those are derived from line intensity ratios. More recent calculations by Troscompt et al. (2009) are more accurate, however, include a more limited range of temperatures and transitions, therefore, were not used in our calculations.

Figure A.1 shows the calculated line ratios as a function of kinetic temperature and $\mathrm{H}_{2}$ density. In the central $40^{\prime \prime} \times 40^{\prime \prime}$ region, $F W H M=12 \mathrm{~km} \mathrm{~s}^{-1}$ was used as an initial parameter for Radex, while outside of this region we used $F W H M=6 \mathrm{~km} \mathrm{~s}^{-1}$, based on the observed values. However, there is no strong dependence on the FWHM of the lines. We apply a molecular column density of $\mathrm{N}\left(\mathrm{H}_{2} \mathrm{CO}\right)=5 \times 10^{14} \mathrm{~cm}^{-2}$ as an "average" value for all the positions over the SLS field. This is slightly below the value that can reproduce the optical depths derived from rotation diagram method for the central position, assuming uniform beam filling.

The results are shown in Fig. 5 with the main regions highlighted. We derive a kinetic temperature of $300 \mathrm{~K}$ toward the center, $98 \mathrm{~K}$ toward the northern clump, $85 \mathrm{~K}$ toward the southwest clump and $68 \mathrm{~K}$ toward the eastern tail, with an uncertainty of $\sim 30 \%$. In fact, there is a large, about $1^{\prime} \times 1^{\prime}(\sim 3 \times 3 \mathrm{pc})$ region around the center, with kinetic temperatures $\gtrsim 100 \mathrm{~K}$.

\subsection{Volume- and column density estimates}

To estimate $\mathrm{H}_{2}$ volume densities of the high temperature gas that the $\mathrm{H}_{2} \mathrm{CO}$ line ratios trace, we use line ratios of the $\mathrm{HCN} 3-2$ (265.886 GHz) and $\mathrm{HCN} 4-3(354.505 \mathrm{GHz})$ transitions. Our calculations are based on collisional rates from Dumouchel et al. (2010), which have been scaled by a factor 1.37 to represent collisions with $\mathrm{H}_{2}$. The $\mathrm{HCN}$ 4-3 map has been convolved to the resolution of the HCN 3-2 map (20"). Since the line ratios depend on the kinetic temperature (Fig. A.2), we use the kinetic temperatures determined from the $\mathrm{H}_{2} \mathrm{CO}$ line ratios to convert the measured $\mathrm{HCN}$ line ratios (Fig. 6) to volume densities. Therefore, even if $\mathrm{HCN}$ was detected towards almost the entire SLS field, our calculations only take into account the positions where both of the $\mathrm{H}_{2} \mathrm{CO}$ transitions were detected. The uncertainty of these density estimates is $\sim$ a factor of two. Figure 7 shows the estimated volume densities. Note that not all the positions with kinetic temperature estimates are included in the density map: this is due to the uncertainty of our method below $100 \mathrm{~K}$, where the HCN 3-2/4-3 line ratios are rather temperature than density tracers. With a search range in the densities between $10^{3}$ and $10^{8} \mathrm{~cm}^{-3}$, an extended area towards the center (approximately corresponding to the high-excitation area revealed by the $\mathrm{H}_{2} \mathrm{CO}$ line ratios) corresponds to densities as high as $10^{5} \mathrm{~cm}^{-3}$ or higher, with a maximum of $6.9 \times 10^{6} \mathrm{~cm}^{-3}$. The maximum density is measured toward an offset position compared to the position we refer to as "center". The densities toward the central part show little less than an order of magnitude variation. 


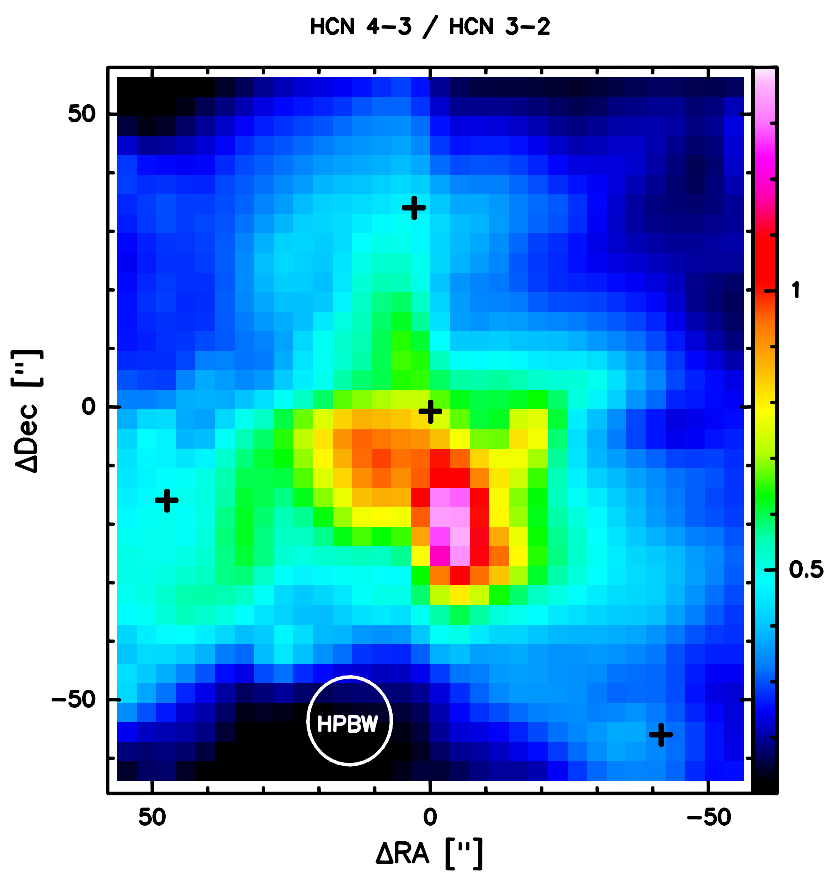

Fig. 6. Map of the line ratio of $\mathrm{HCN} J=4-3$ to $\mathrm{HCN} J=3-2$.

The average $\mathrm{H}_{2}$ volume density corresponding to the central $1 \times 1$ arcmin region is $1.6 \times 10^{6} \mathrm{~cm}^{-3}$. The lowest densities, toward the edges of the cloud area covered by our estimates, is a few times $10^{4} \mathrm{~cm}^{-3}$. However, our density estimates are more accurate for densities between $10^{5}$ and $10^{8} \mathrm{~cm}^{-3}$ (Fig. A.2). In addition to the uncertainty of the method below $\sim 10^{5} \mathrm{~cm}^{-3}$, the $\mathrm{H}_{2} \mathrm{CO} 5_{15}-4_{14}$ and $5_{33}-4_{32}$ transitions are detected over a less extended region, compared to the $\mathrm{HCN} 3-2$ and 4-3 transitions. Estimating the physical conditions outside the area covered by our kinetic temperature and volume density maps requires molecular line tracers with a larger spatial extent.

Based on the kinetic temperatures and volume densities presented above, we estimate the column densities of the two main molecular tracers used in this paper, $\mathrm{HCN}$ and $\mathrm{H}_{2} \mathrm{CO}$. These estimates use $\mathrm{H}_{2}$ volume densities of $1.8 \times 10^{6} \mathrm{~cm}^{-3}$ for the center, $1.2 \times 10^{6} \mathrm{~cm}^{-3}$ for the eastern tail, $5.6 \times 10^{5} \mathrm{~cm}^{-3}$ for the northern clump and $1.3 \times 10^{5} \mathrm{~cm}^{-3}$ for the southwest clump. The kinetic temperatures used for each position are listed in Sect. 3.3. To calculate the HCN column density toward the center, we use the $\mathrm{H}^{13} \mathrm{CN} 4-3(345.3 \mathrm{GHz})$ transition, and ${ }^{12} \mathrm{C} /{ }^{13} \mathrm{C}=77$ (Wilson \& Rood 1994). HCN peaks toward the center, with a column density of $7.3 \times 10^{15} \mathrm{~cm}^{-2}$. The HCN column densities of the eastern tail and northern clump regions are comparable: $N(\mathrm{HCN})=7-8 \times 10^{13} \mathrm{~cm}^{-2}$ toward the eastern tail and $N(\mathrm{HCN})=1.5-2 \times 10^{14} \mathrm{~cm}^{-2}$ toward the northern clump. $N(\mathrm{HCN})$ toward the southwest clump is a factor of $4-5$ above the column density of the northern clump: $N(\mathrm{HCN})=6.2-9.6 \times$ $10^{14} \mathrm{~cm}^{-2} . N\left(\mathrm{H}_{2} \mathrm{CO}\right)$ is $\sim 3.2 \times 10^{14}-4.5 \times 10^{14} \mathrm{~cm}^{-2}$ toward the center as well as the southwest clump. The northern clump has $N\left(\mathrm{H}_{2} \mathrm{CO}\right)$ slightly below the center and southwest clump, $7.0 \times 10^{13}-1.1 \times 10^{14} \mathrm{~cm}^{-2}$, while toward the eastern tail, we measure a column density of $4.9 \times 10^{13}-5.0 \times 10^{13} \mathrm{~cm}^{-2}$. The two orders of magnitude difference in the $\mathrm{H}_{2} \mathrm{CO}$ column density toward the center compared to the best fit column density derived from the rotation diagrams can be accounted for the simple assumption used in the rotation diagram method, that the level populations can be described by a single excitation temperature.

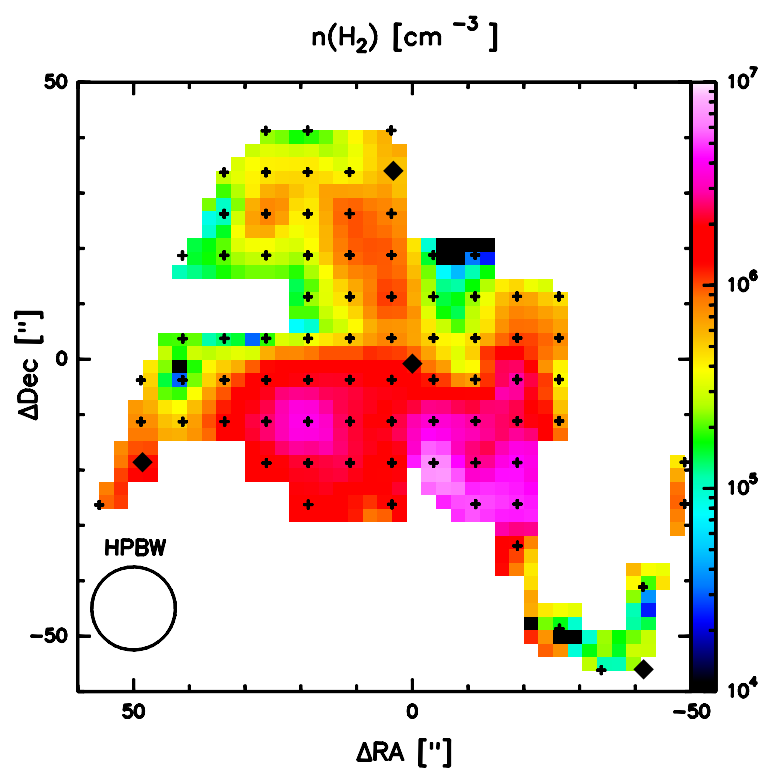

Fig. 7. Volume density estimates from the $\mathrm{HCN} 3-2$ and HCN 4-3 lines.

The mass of the warm and dense gas can be estimated based on the $\mathrm{H}_{2} \mathrm{CO}$ column density, by assuming an $\mathrm{H}_{2} \mathrm{CO}$ abundance. Van der Tak et al. (2000) derive $\mathrm{H}_{2} \mathrm{CO}$ abundances in the range of $10^{-9}-10^{-10}$ for thirteen regions of massive star formation. For an "average" column density of $10^{14} \mathrm{~cm}^{-2}$ and an abundance of $10^{-9}$, the mass of the central $1^{\prime} \times 1^{\prime}$ region with warm and dense gas is $\sim 2 \times 10^{4} M_{\odot}$, which becomes $\sim 2 \times 10^{5} M_{\odot}$ for an $\mathrm{H}_{2} \mathrm{CO}$ abundance of $10^{-10}$. These are comparable to the mass estimates based on dust continuum measurements. WardThompson \& Robson (1990) estimate a mass of $\sim 2.4 \times 10^{5} \mathrm{M}_{\odot}$ corresponding to the central $\sim 2$ pc. Buckley \& Ward-Thompson (1996) adopt a value of $\sim 10^{5} M_{\odot}$ as a best fit based on dust continuum as well as on CS data published by Serabyn et al. (1993).

A second estimate of the mass of dense gas can be obtained from the HCN analysis. Adopting an average number density of $1.6 \times 10^{6} \mathrm{~cm}^{-3}$ over the central $1^{\prime} \times 1^{\prime}$ region and assuming an approximately spherical region would imply a mass of $\sim 2 \times 10^{6} M_{\odot}$. This is a factor of 10-100 larger than the $\mathrm{H}_{2} \mathrm{CO}$ suggests indicating that the dense $\mathrm{HCN}$ material fills a fraction between $1 \%$ and $10 \%$ of the volume of this extended region.

Further evidence for a small volume filling factor is given by the best fit source sizes from the rotation diagrams toward the central position. The column densities derived from Radex are beam-averaged, while the best fit column densities from the rotation diagram correspond to clumps with sizes indicated by the best fit source size. Comparing the HCN column densities to the $\mathrm{H}_{2}$ volume densities gives another indication of the volume filling factor. While the volume densities change between $10^{5}$ and a few times $10^{6} \mathrm{~cm}^{-3}$ and show a rather uniform distribution, $N(\mathrm{HCN})$ shows a 2 orders of magnitude variation $\left(7 \times 10^{13}-7 \times 10^{15} \mathrm{~cm}^{-2}\right)$ and peaks toward the center. The emission may originate in warm and dense gas in a number of unresolved clumps. Such a behavior was found before, by Snell et al. (1984) and Mundy et al. (1986) for M17, S140 and NGC 2024.

\section{Discussion}

We have detected warm $(\gtrsim 100 \mathrm{~K})$ and dense $\left(\gtrsim 10^{5} \mathrm{~cm}^{-3}\right)$ gas extended over a $1^{\prime} \times 1^{\prime}(\sim 3 \times 3 \mathrm{pc})$ field towards W49A. Based on a rotation diagram analysis with corrections for optical depth and beam dilution, we derive excitation temperatures of $115 \mathrm{~K}$ (for 
$\mathrm{SO}_{2}$ ), $82 \mathrm{~K}$ (for $\mathrm{CH}_{3} \mathrm{OH}$ ), $138 \mathrm{~K}$ (for $\mathrm{H}_{2} \mathrm{CO}$ ) towards the center. From $\mathrm{H}_{2} \mathrm{CO}$ line ratios with the same $J$ and different $K$-states, we derive kinetic temperatures of $\sim 300 \mathrm{~K}$ toward the center, $68 \mathrm{~K}$ toward the eastern tail, $98 \mathrm{~K}$ toward the northern clump and $85 \mathrm{~K}$ toward the southwest clump. Our kinetic temperature estimates are consistent with the excitation temperatures derived from the rotation diagram method, given that non-LTE conditions are expected, with excitation temperatures below the kinetic temperatures. Ratios of HCN 3-2 / HCN 4-3 line intensities, using our kinetic temperature estimates, result in $\mathrm{H}_{2}$ volume densities of $1.8 \times 10^{6} \mathrm{~cm}^{-3}$ for the center, $1.2 \times 10^{6} \mathrm{~cm}^{-3}$ for the eastern tail, $5.6 \times 10^{5} \mathrm{~cm}^{-3}$ for the northern clump and $1.3 \times 10^{5} \mathrm{~cm}^{-3}$ for the southwest clump.

Our kinetic temperature estimates are significantly higher than previously reported values. Based on Planck brightness temperatures derived from the $\mathrm{CO} J=7-6$ transition, Jaffe et al. (1987) estimate kinetic temperatures of $>70 \mathrm{~K}$ in the centre, falling below $50 \mathrm{~K}$ in the outer parts of the cloud. However, these observations have a beam size of $32^{\prime \prime}$, which suggests higher kinetic temperatures on a smaller scale, and as such, may be consistent with our results. Roberts et al. (2011) use HCN/HNC line ratios compared to chemical models to estimate kinetic temperatures and derive temperatures of $100 \mathrm{~K}$ toward the center, $40 \mathrm{~K}$ toward the southwest clump, $40 \mathrm{~K}$ toward the eastern tail and $75 \mathrm{~K}$ toward the northern clump. One possible reason of the difference between the kinetic temperatures presented in this paper and the values estimated by Roberts et al. (2011) is that Roberts et al. (2011) adopted the HCN collision rates for HNC, which can result in an uncertainty, due to the fact that HCN and HNC have slightly different dipole moments (3.0 vs. $3.3 \mathrm{D})$.

Our density estimates are consistent with the estimates by Welch et al. (1987), who estimate the cloud density to be $>3 \times 10^{5} \mathrm{~cm}^{-3}$ within the inner $1 \mathrm{pc}$ and $\sim 10^{4} \mathrm{~cm}^{-3}$ in the outer parts of the cloud. Our estimates are also consistent with (Plume et al. 1997), who find - based on CS 2-1, 3-2, 5-4, 14-13, 10-9 transitions - that $n\left(\mathrm{H}_{2}\right)$ toward W49A varies between $5.2 \times 10^{5}$ and $1.8 \times 10^{6} \mathrm{~cm}^{-3}$. Serabyn et al. (1993) estimate densities between $2-6 \times 10^{6} \mathrm{~cm}^{-3}$ for three clumps using CS $(3-2,5-4,7-6,10-9)$ and $C^{34} S(5-4,7-6,10-9)$ transitions. Our mass, volume- and column density estimates also indicate a low filling factor for the dense gas. Plume et al. (1997) probe masses and densities for a number of massive star-forming regions, and find, that filling factor for the dense gas is $<25 \%$.

\subsection{Possible heating sources}

The most probable mechanisms that contribute to the heating of the warm and dense molecular gas seen toward W49A are mechanical heating (shocks produced by stellar winds), and UV or X-ray irradiation. Another possible mechanism, that has been suggested for starburst galaxies, is heating by cosmic rays (Bradford et al. 2003). In the case of W49A, the only possible source of cosmic-rays above the Galactic background is the supernova remnant W49B, however, due to the high uncertainty in the distance of W49B (Brogan \& Troland 2001), this remains an open question. Therefore, we focus on quantifying the effect of mechanical heating and irradiation by UV and X-rays for the warm and dense region revealed by our $\mathrm{H}_{2} \mathrm{CO}$ and $\mathrm{HCN}$ data.

Mechanical heating. The luminosity corresponding to the mechanical heating produced by stellar winds of embedded O-type stars can be estimated from:

$L_{\mathrm{w}}=1.3 \times 10^{36}\left(\frac{\dot{M}}{10^{-6} M_{\odot} \text { year }^{-1}}\right)\left(\frac{v_{\mathrm{w}}}{2 \times 10^{3} \mathrm{~km} \mathrm{~s}^{-1}}\right)^{2} \mathrm{erg} \mathrm{s}^{-1}$ where $v_{\mathrm{w}}=2000 \mathrm{~km} \mathrm{~s}^{-1}$ can be applied as the velocity of the stellar wind (Tielens 2005). Peng et al. (2010) found evidence for two expanding shells with a size of $\sim 2.9 \mathrm{pc}$, comparable to the size of the extended high excitation region we have found. They found that a constant mass-loss rate of $\sim 1.2 \times 10^{-6} M_{\odot} \mathrm{yr}^{-1}$ can sustain a wind-driven bubble with a size of $\sim 2.9 \mathrm{pc}$, which corresponds to the mass loss rate of one O-type star. Using these values, the mechanical luminosity is $L_{\mathrm{w}}=1.56 \times 10^{36} \mathrm{erg} \mathrm{s}^{-1}$ or about $405 L_{\odot}$. Assuming an efficiency of $1-10 \%$ (Loenen et al. 2008), the mechanical heating rate is $\sim 4-40.5 L_{\odot}$. However, this is only a lower limit of the luminosity corresponding to the mechanical heating. Alves \& Homeier (2003) estimate that 30 Otype stars belong to the central stellar cluster, which has a diameter of $\sim 6 \mathrm{pc}$. Taking this into account, the mass-loss rate, as well as the luminosity is about an order of magnitude larger than the value we derive by taking a mass-loss rate that belongs to one O-type star. This gives $40.5-405 L_{\odot}$ as a mechanical heating luminosity.

In addition to the effect of stellar winds, protostellar outflows give a contribution to the mechanical heating. The largest outflow is connected to the most luminous water-maser emission in the Milky Way (Gwinn et al. 1992). It has been characterized by Scoville et al. (1986) and recently by Smith et al. (2009). Adopting an outflow dynamical time-scale of $10^{4} \mathrm{yr}$, that corresponds to an average $\sim 25 \mathrm{~km} \mathrm{~s}^{-1}$ outflow speed (Smith et al. 2009), an energy of $4.4 \times 10^{47} \mathrm{erg}$ (Scoville et al. 1986, scaled to a distance of $11.4 \mathrm{kpc}$ ), the mechanical luminosity is $L_{\text {mech }}=1.4 \times 10^{36} \mathrm{erg} \mathrm{s}^{-1}$ or about $\sim 400 L_{\odot}$. Assuming the same efficiency as for the mechanical luminosity produced by the stellar wind, only the most powerful outflow produces a mechanical luminosity that is $10 \%$ of the mechanical luminosity produced by the stellar winds of an embedded cluster of $\sim 30$ O-type stars. This estimate gives a lower limit on the mechanical luminosity produced by outflows. Additional kinetic energy injection may have been resulted by past outflows of the current O-type stars. These flows may still be dissipating energy. The existence of these flows may be probed using ALMA.

An additional source of the mechanical heating may exist in the form of shocks driven by the expansion of the (UC)HII regions into the molecular cloud. The corresponding heating rate is proportional to the kinetic energy injection rate and is given by:

$G=\frac{1}{2} A \frac{\mathrm{d} \rho}{\mathrm{d} t} V^{2}=\frac{1}{2} \rho A V^{3}$

where $A$ is the surface area of the shocks, $\rho$ is the density of the medium into which the shock is moving, and $V$ is the velocity of the shock. The surface area of the shocks can be estimated based on De Pree et al. (1997), who measure the sizes of the (UC)HII regions, based on high-resolution ( $\left.00^{\prime} 8\right) 3.6 \mathrm{~cm}$ data. We use an average radius of $0.05 \mathrm{pc}$ to calculate the surface area of the shocks. Based on $n\left(\mathrm{H}_{2}\right)=10^{6} \mathrm{~cm}^{-3}$, a shock velocity of $10 \mathrm{~km} \mathrm{~s}^{-1}$ results in a heating rate of $1.6 \times 10^{37} \mathrm{erg} \mathrm{s}^{-1}$ for a cluster of 30 O-type stars. For a shock velocity of $5 \mathrm{~km} \mathrm{~s}^{-1}$, the mechanical heating rate of the expanding (UC)H $\mathrm{H}$ regions corresponding to the cluster of 30 O-type stars is $\sim 2 \times 10^{36} \mathrm{erg} \mathrm{s}^{-1}$ - in the same order of magnitude as the mechanical heating produced by the stellar winds of the 30 O-type stars.

Radiative heating by the embedded stellar cluster. The importance of heating by $U V$ radiation from young massive stars, in the form of photo-electric emission by dust grains and PAHs, can be estimated from the far-infrared luminosity. Vastel et al. (2001) estimates a total FIR flux of $1.54 \times 10^{-6} \mathrm{erg} \mathrm{s}^{-1} \mathrm{~cm}^{-2}$, 
which, at the distance of W49A, is equivalent to a total IR luminosity of $6.3 \times 10^{6} L_{\odot}$. Adopting an efficiency of $10^{-3}-10^{-2}$ (Meijerink \& Spaans 2005), the heating rate by UV irradiation is on the same order of magnitude as the luminosity that can be expected from the mechanical heating.

$X$-ray heating. Hard X-ray emission has been detected (Tsujimoto et al. 2006) toward the center of W49A, associated with two Hir regions. They measure an X-ray luminosity of $3 \times 10^{33} \mathrm{erg} \mathrm{s}^{-1}$ in the $3-8 \mathrm{keV}$ band. Assuming an efficiency of $10 \%$ (Meijerink \& Spaans 2005), the X-ray heating luminosity is on the order of $0.1 L_{\odot}$, about three orders of magnitudes below the luminosity of the mechanical and UV heating. In addition, since X-ray emission is due to point-sources, rather than an extended region, X-rays mostly act locally and seem less likely to be responsible for the extended emission region what we detected.

Gas-grain collisional heating. At the densities estimated using HCN transitions $\left(10^{5}-10^{6} \mathrm{~cm}^{-3}\right)$, gas-grain collisions may give a significant contribution to the heating of the gas. Based on a greybody fit to the dust emission, Ward-Thompson \& Robson (1990) derive a dust temperature of $50 \mathrm{~K}$. They also find evidence, that a population of small, hot dust grains with $T \sim 350 \mathrm{~K}$ also exists, based on an excess in the near-infrared. The heating rate of the gas per unit volume can be estimated using (Hollenbach \& McKee 1979, 1989):

$$
\begin{aligned}
\Gamma_{\text {coll. }}= & 1.2 \times 10^{-31} n^{2}\left(\frac{T_{\text {kin }}}{1000}\right)^{1 / 2}\left(\frac{100 \AA}{a_{\text {min }}}\right)^{1 / 2} \\
& \times\left[1-0.8 \exp \left(-75 / T_{\text {kin }}\right)\right]\left(T_{\mathrm{d}}-T_{\text {kin }}\right)\left(\mathrm{erg} \mathrm{cm}^{-3} \mathrm{~s}^{-1}\right)
\end{aligned}
$$

where the minimum grain size is set at $a_{\min }=10 \AA$. Using $T \sim$ $150 \mathrm{~K}$ and $n=10^{6} \mathrm{~cm}^{-3}$, assuming a volume filling factor of $1-10 \%$ for the hot gas, a gas-to-dust ratio of 100 , and that the fraction of the warm $(T \sim 350 \mathrm{~K})$ dust is up to $1 \%$ of the total dust, gas-grain heating for a $3 \times 3 \mathrm{pc}$ region toward the center of W49A is expected to be $\sim 10^{34}-10^{35} \mathrm{erg} \mathrm{s}^{-1}$, equivalent to $\sim 3-$ $30 L_{\odot}$.

Based on these estimates, mechanical heating and UV heating seem to be the most probable heating mechanisms, while irradiation by X-rays and gas-grain collisions are less probable. The contribution of radiation to the excitation of W49A has been investigated earlier by Roberts et al. (2011), based on HCN, $\mathrm{HNC}$ and $\mathrm{HCO}^{+}$transitions from the SLS. By comparing the observed line intensity ratios to PDR (Photon-dominated Region) and XDR (X-ray Dominated Region) models of Meijerink et al. (2007), they find, that $\mathrm{HCN} / \mathrm{HCO}^{+}$line ratios are consistent with PDR models, while HCN/HNC line ratios are consistent with XDR models. They interpret this result as evidence that irradiation by UV and X-ray photons plays a minor role in the excitation of W49A. However, as seen above, based on the UV heating luminosity, UV radiation cannot be ruled out to contribute significantly to the heating of W49A.

\subsection{Comparison to the cooling rate}

One of the main contributions to the total cooling in W49A is expected to occur via $\mathrm{CO}$ lines, therefore we derive a lower limit on the total cooling rate from the ${ }^{12} \mathrm{CO} 3-2(345.8 \mathrm{GHz})$ line luminosity based on data from the SLS. The CO line luminosity can be calculated from the CO 3-2 line intensities (Solomon \& Vanden Bout 2005), as

$L_{\mathrm{CO}}\left[\mathrm{K} \mathrm{km} \mathrm{s}^{-1} \mathrm{pc}^{2}\right]=23.5 \Omega\left[\operatorname{arcsec}^{2}\right](d[\mathrm{Mpc}])^{2} I_{\mathrm{CO}}\left[\mathrm{K} \mathrm{km} \mathrm{s}^{-1}\right]$ or alternatively, in units of $L_{\odot}$ :

$$
L_{\mathrm{CO}}\left[L_{\odot}\right]=1.04 \times 10^{-3} S_{\mathrm{CO}}[\mathrm{Jy}] \Delta V\left[\mathrm{~km} \mathrm{~s}^{-1}\right] v[\mathrm{GHz}](d[\mathrm{Mpc}])^{2}
$$

where $I_{\mathrm{CO}}=593.4 \mathrm{~K} \mathrm{~km} \mathrm{~s}^{-1}, T_{\mathrm{B}}=34.5 \mathrm{~K}, S_{\mathrm{CO}}=1.2 \times 10^{4} \mathrm{Jy}$ (using the Rayleigh-Jeans law to convert the brightness temperature to flux density) and $\Delta V=17.2 \mathrm{~km} \mathrm{~s}^{-1}$ is the average line width. These numbers correspond to a spectrum smoothed to a $F W H M=1^{\prime}$ beam, which covers the high-excitation region around the center. Using these numbers, $L_{\mathrm{CO}(3-2)}=6.5 \times$ $10^{3} \mathrm{~K} \mathrm{~km} \mathrm{~s}^{-1} \mathrm{pc}^{2}=9.65 L_{\odot}$ for the highly excited central square arcminute region. The fraction of the flux of the $\mathrm{CO} 3-2$ transition to the total $\mathrm{CO}$ line flux can be estimated using Radex, adopting an $\mathrm{H}_{2}$ volume density of $10^{6} \mathrm{~cm}^{-3}$ and a $\mathrm{CO}$ column density of $10^{19} \mathrm{~cm}^{-2}$. The $\mathrm{CO}$ column density estimate is based on the $\mathrm{C}^{17} \mathrm{O} 3-2$ transition (at $\sim 337 \mathrm{GHz}$ ) and a ratio of ${ }^{16} \mathrm{O} /{ }^{17} \mathrm{O} \sim 1800$ (Wilson \& Rood 1994). For $T_{\text {kin }}=100 \mathrm{~K}$, the 3-2 transition is expected to be $9 \%$, and for $T_{\text {kin }}=300 \mathrm{~K}, 5 \%$ of the total CO line flux. Using our Radex estimates of the total CO line flux:

$$
\begin{aligned}
L_{\mathrm{CO}} & =7.2 \times 10^{4}-1.3 \times 10^{5} \mathrm{~K} \mathrm{~km} \mathrm{~s}^{-1} \mathrm{pc}^{2} \\
& =107.2-192.9 L_{\odot} \\
& =4.1 \times 10^{35}-7.4 \times 10^{35} \mathrm{erg} \mathrm{s}^{-1},
\end{aligned}
$$

gives a lower limit on the cooling, with an accuracy of $\sim 20 \%$, mainly as a contribution from the typical calibration error in deriving the $\mathrm{CO} 3-2$ line intensity.

Based on Neufeld et al. (2005), for kinetic temperatures $>100 \mathrm{~K}$ and $\mathrm{H}_{2}$ volume densities $\sim 10^{6} \mathrm{~cm}^{-3}$, the total cooling is up to an order of magnitude above the CO cooling, $\sim 10^{3} L_{\odot}$.

Our estimates of the heating rate indicate that the two most important mechanisms to consider for W49A, are mechanical heating by the winds of O-type stars and UV-irradiation. These two mechanisms give a heating rate equivalent with $1000 L_{\odot}$, depending on the heating efficiency. This is of a same order of magnitude as the expected total cooling, assuming that the total cooling rate is a factor of 10 higher than the $\mathrm{CO}$ cooling rate. This result supports that the effect of embedded O-type stars in the form of stellar winds and UV-irradiation can explain the high-excitation region around the center of W49A.

\subsection{Comparison to other warm and dense regions}

Galactic center. In our Galaxy, a similar level of excitation to what we found toward W49A was reported for clouds near the center. Hüttemeister et al. (1993) measured kinetic temperatures in 36 clouds near the Galactic center and based on $\mathrm{NH}_{3}$ transitions, they have found a temperature component of $\sim 200 \mathrm{~K}$, together with a cold gas and dust component. This sample, however, include no embedded stars, which could explain the highly excited gas component, and therefore, cannot be used as an analogue for W49A. De Vicente et al. (1997) have detected hot molecular gas toward the Sagittarius B2 molecular cloud. The highest temperatures measured toward Sagittarius B2 are in the range between 200-400 K, with typical densities of $10^{6}-10^{7} \mathrm{~cm}^{-3}$, similar to what we find for the center of W49A. However, these conditions correspond to hot cores with sizes in the range of $0.5-0.7 \mathrm{pc}$, much smaller than the region with similar physical properties in W49A. De Vicente et al. (1997) have also found a more extended component of warm (100-120 K) gas as a ring surrounding Sgr B2M and Sgr B2N with a radius of $2 \mathrm{pc}$ and a thickness of $1.4 \mathrm{pc}$, with a density of about 
$2 \times 10^{5} \mathrm{~cm}^{-3}$. Ferrière et al. (2007) study physical conditions in the innermost $3 \mathrm{kpc}$ of the Galaxy, and find a high-temperature gas component ( $T \sim 150 \mathrm{~K})$, however, it corresponds to a lowdensity environment $\left(n_{\mathrm{H}_{2}} \sim 10^{2.5} \mathrm{~cm}^{-3}\right)$, unlike what we find for W49A. On Galactic scales, W49A shows a uniquely extended region of high $(T \gtrsim 100 \mathrm{~K})$ temperature which appears to be tracing the feedback from the young and forming stars in the region on their surrounding gas.

Starbursts and active galaxies. In even nearby galaxies sensitivity and angular resolution mean that the physical conditions of the molecular gas can only be estimated on $\sim 100 \mathrm{pc}$ scales with uncertain filling factors. Nevertheless there are regions where temperatures similar to those in W49A are measured. In particular, extended warm molecular gas $(>100 \mathrm{~K})$ has been detected in a number of starburst- and Seyfert galaxies (e.g. Rigopoulou et al. 2002). Mühle et al. (2007) have detected a warm, $T_{\text {kin }} \sim 200 \mathrm{~K}$ gas component with moderate density $\left(n\left(\mathrm{H}_{2}\right)=7 \times 10^{3} \mathrm{~cm}^{-3}\right)$ in the starburst galaxy M82, using $\mathrm{p}-\mathrm{H}_{2} \mathrm{CO}$ line ratios, probing scales of $\sim 320 \mathrm{pc}$. Ott et al. (2005) derived kinetic temperatures of $140 \mathrm{~K}$ and $200 \mathrm{~K}$ toward two positions of the starburst galaxy NGC 253, using $\mathrm{NH}_{3}(3,3)$ ATCA data on a scale of 65 pc. Mauersberger et al. (2003) have detected a warm (100-140 K) gas component toward the center of three nearby starburst galaxies (NGC 253, IC 342 and Maffei 2) for regions of 300-660 pc, based on $\mathrm{NH}_{3}(1,1)-(6,6)$ transitions. An even warmer component $(T>400 \mathrm{~K})$ was detected toward IC 342. Papadopoulos et al. (2012), based on a sample of 70 (Ultra-)Luminous Infrared Galaxies, most of them dominated by a warm $\left(T_{\text {kin }}>100 \mathrm{~K}\right)$ and dense $\left(n>10^{4} \mathrm{~cm}^{-3}\right)$ gas phase with masses of $\sim 10^{9} M_{\odot}$, come to the conclusion that the main heating mechanism is rather cosmic-rays and highly supersonic turbulence than far-UV radiation or shocks related to starformation. This might be one of the key differences between the extended, but compared to scales of entire galaxies, still local phenomenon seen for W49A, and starburst galaxies. However, in the context of the Milky Way, W49A is a close analogue in terms of physical parameters to regions in starburst- and active galaxies.

Another key comparison with warm $(\gtrsim 100 \mathrm{~K})$ and dense $\left(\gtrsim 10^{5} \mathrm{~cm}^{-3}\right)$ regions, such as starburst galaxies, is the initial mass function (IMF). Klessen et al. (2007) have found, based on hydrodynamical numerical calculations, that for regions of warm $(\sim 100 \mathrm{~K})$ and dense $\left(\gtrsim 10^{5} \mathrm{~cm}^{-3}\right)$ gas, the slope of the IMF is in the range of -1.0 and -1.3 , with a turn-over mass of $7 M_{\odot}$. Homeier \& Alves (2005) derived an IMF in W49A with a slope of $-1.70 \pm 0.30$ and $-1.6 \pm 0.3$ down to masses of $\sim 10 M_{\odot}$. This result may be consistent with the calculations of Klessen et al. (2007), and if so, supports that W49A is a Galactic template for starburst galaxies. However, incompleteness at low luminosities and masses might explain the observed cut-off at masses of $\sim 10 M_{\odot}$.

Pressure as a diagnostic of starburst environments. Environments with increased star-formation activity, such as starburst galaxies, massive star-forming regions and clouds near the Galactic center can be characterized with their pressures, $P / k \sim n T$. Typical pressures that can be expected in the Galaxy and in the midplane of ordinary spirals are in the order of $10^{4} \mathrm{~K} \mathrm{~cm}^{-3}$ (Papadopoulos et al. 2012). Table 4 shows the measured values of $n, T$ and $P / k$ for W49A, Sgr B2 and starburst galaxies. $P / k$ values show more than an order of magnitude variation. Based on our derived densities and temperatures, the gas
Table 4. Comparison of physical parameters in W49A to Galactic center clouds and starburst galaxies.

\begin{tabular}{l|ccc}
\hline \hline Source & $\begin{array}{c}n \\
\left(\mathrm{~cm}^{-3}\right)\end{array}$ & $\begin{array}{c}T \\
(\mathrm{~K})\end{array}$ & $\begin{array}{c}P / k \\
\left(\mathrm{~K} \mathrm{~cm}^{-3}\right)\end{array}$ \\
\hline W49A center & $1.8 \times 10^{6}$ & 300 & $5.4 \times 10^{8}$ \\
W49A N clump & $5.6 \times 10^{5}$ & 98 & $5.5 \times 10^{7}$ \\
W49A E tail & $1.2 \times 10^{6}$ & 68 & $8.2 \times 10^{7}$ \\
W49A SW clump & $1.3 \times 10^{5}$ & 85 & $1.1 \times 10^{7}$ \\
\hline Sgr B2 $^{a}$ & $2 \times 10^{5}$ & $100-120$ & $2 \times 10^{7}-2.4 \times 10^{7}$ \\
Henize 2-10 $^{b}$ & $\gtrsim 1 \times 10^{4}$ & $50-100$ & $5 \times 10^{5}-10^{6}$ \\
M82 & $10^{4.2}-10^{4.4}$ & $130-160$ & $10^{6.4}-10^{6.6}$ \\
Arp 220 & $(0.3-1) \times 10^{6}$ & $45-120$ & $1.4 \times 10^{7}-1.2 \times 10^{8}$ \\
Antennae $^{e}$ & $10^{5}$ & 90 & $9 \times 10^{6}$ \\
\hline
\end{tabular}

Notes. ${ }^{(a)}$ De Vicente et al. (1997) based on $\mathrm{CH}_{3} \mathrm{CN} J=5-4,8-7$, 12-11 lines. ${ }^{(b)}$ Bayet et al. (2004), based on ${ }^{12} \mathrm{CO}(J=3-2,4-3,6-5$, $7-6)$ and ${ }^{13} \mathrm{CO}(J=3-2)$. ${ }^{(c)}$ Median values based on three different positions (Naylor et al. 2010). Obtained from multiple species radiative transfer modeling of the lines of $\mathrm{CS}, \mathrm{HCO}^{+}, \mathrm{HCN}, \mathrm{HNC}$, and $\mathrm{C}^{34} \mathrm{~S}$. ${ }^{(d)}$ Greve et al. (2009), based on HCN and CS lines. ${ }^{(e)}$ Zhu et al. (2003), based on $\mathrm{CO}$ and ${ }^{13} \mathrm{CO} 2-1$ and 3-2 lines.

pressure in W49A is up to $5.4 \times 10^{8} \mathrm{~K} \mathrm{~cm}^{-3}$, similar to those measured toward SgrB2 and Arp 220. Other galactic nuclei appear to have lower pressures, which seems to be mainly the effect of a lower gas density. Beam dilution seems not to play a major effect for the density estimates, given the high densities measured toward Arp 220 as well as the sensitivity, which makes measurements in external galaxies biased toward the warm and dense gas. However, the estimated densities significantly depend on the tracer used, as was shown by Greve et al. (2009), who find a 10-100 factor difference between densities estimated from $\mathrm{HCN}$ compared to densities estimated from $\mathrm{HCO}^{+}$. Therefore, the uncertainty in $P / k$ is about an order of magnitude.

Pressures measured toward W49A and Sgr B2 are not entirely unique in our Galaxy. Dense gas has been detected toward a number of massive star forming regions, such as reported by Plume et al. (1997), Wu et al. (2010) and McCauley et al. (2011). However, the temperatures of those regions are only comparable to what we measure toward W49A "locally", on sub-parsec scales, corresponding to hot cores, such as toward Orion KL (Mangum \& Wootten 1993). Considering the spatial extent, pressures measured toward W49A provide a Galactic analogue of starburst galaxies.

\section{Summary and conclusions}

We have analyzed the physical conditions towards the central $2 \times 2$ arcminutes region of W49A using JCMT maps with $15^{\prime \prime}$ resolution. We have reported a detection of warm $(>100 \mathrm{~K})$ and dense $\left(>10^{5} \mathrm{~cm}^{-3}\right)$ gas toward the center, extending up to a few parsecs.

- We have characterized the excitation toward four main regions of W49A (center, northern clump, southwest clump and eastern tail) based on rotational diagrams of $\mathrm{SO}_{2}$, $\mathrm{CH}_{3} \mathrm{OH}$ and $\mathrm{H}_{2} \mathrm{CO}$, taking into account the optical depth and beam dilution. High excitation and column densities, as well as significant beam dilution is seen towards the center, likely to trace embedded hot cores. The off-center positions show excitation temperatures corresponding to about half of those derived for the center, and no significant beam dilution. 


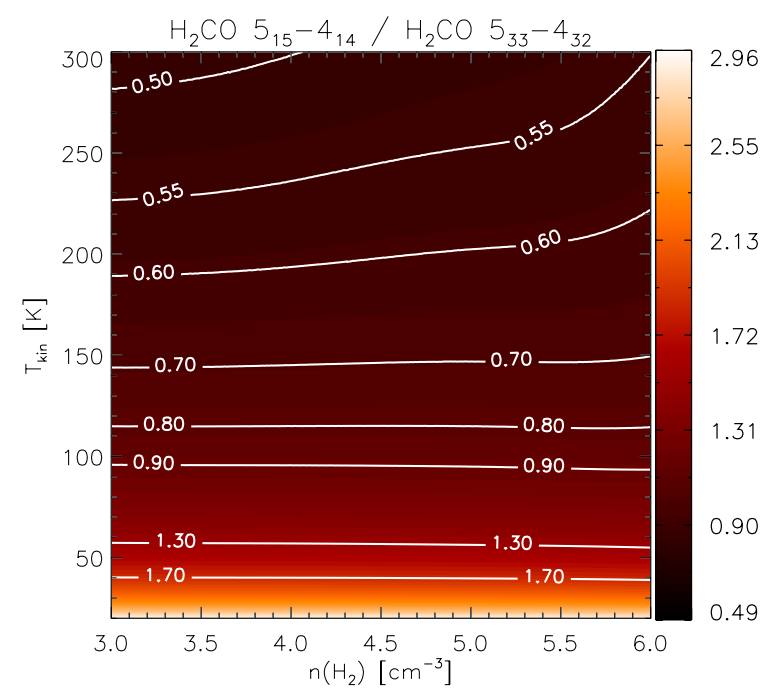

Fig. A.1. Line ratios of o- $\mathrm{H}_{2} \mathrm{CO} 5_{15}-4_{14}$ and $5_{33}-4_{32}$ as a function of kinetic temperature and $\mathrm{H}_{2}$ density for $F W H M=12 \mathrm{~km} \mathrm{~s}^{-1}$. The intensity scale is logarithmic.

- Based on $\mathrm{o}-\mathrm{H}_{2} \mathrm{CO}$ line ratios, we estimate kinetic temperatures of the main regions of W49A. Toward the center, we derive a kinetic temperature of 250-300 K, 60-100 K toward the northern clump, $80-150 \mathrm{~K}$ toward the southwest clump and 70-130 K toward the eastern tail. Our estimates show an approximately $1^{\prime} \times 1^{\prime}(\sim 3.3 \times 3.3 \mathrm{pc})$ region with extended warm $(>100 \mathrm{~K})$ gas towards the center of W49A. Based on HCN 3-2 / HCN 4-3 line ratios, the high-temperature central $1^{\prime} \times 1^{\prime}$ region has $\mathrm{H}_{2}$ volume densities of $>10^{5} \mathrm{~cm}^{-3}$. The high excitation of W49A is comparable to clouds near the center of our Galaxy and to starburst galaxies with estimates on scales of $\sim 100 \mathrm{pc}$.

- The most important heating mechanisms for W49A are mechanical heating due to stellar winds of embedded, O-type stars and UV irradiation. X-ray irradiation and gas-grain collisional heating are less probable than other two mechanisms, while the effect of cosmic-rays remains an open question. However, the two main mechanisms corresponding to embedded young stellar populations provide a heating that no other mechanism is needed to contribute significantly in the heating of W49A. Based on earlier studies of starburst galaxies and their proposed heating mechanisms, W49A is a Galactic starburst analogue, in terms of excitation and physical conditions, but not in terms of the heating mechanism which yields the high excitation, due to additional heating mechanisms that have been proposed to explain the excitation of starburst- and active galaxies (such as cosmic rays or supersonic turbulence).

Our future work includes an analysis of the chemical inventory as well as kinematics of selected species, based on the entire SLS survey including data from the main survey (330-360 GHz, Plume et al. 2007) and will be presented in Nagy et al. (in prep.).

Acknowledgements. We thank the referees John Bally and Adam Ginsburg for the careful reading of the manuscript and for the constructive suggestions. We also thank the editor Malcolm Walmsley for additional helpful comments. The authors thank Alexandre Faure for discussion on $\mathrm{H}_{2} \mathrm{CO}$ collision rates, KuoSong Wang for advice about population diagrams and Paul Goldsmith for discussion about the cooling rates and volume filling factors. Z.N. acknowledges Stefanie Mühle and Chris dePree for their useful comments. We acknowledge the use of the CDMS and JPL databases for the rest frequencies.

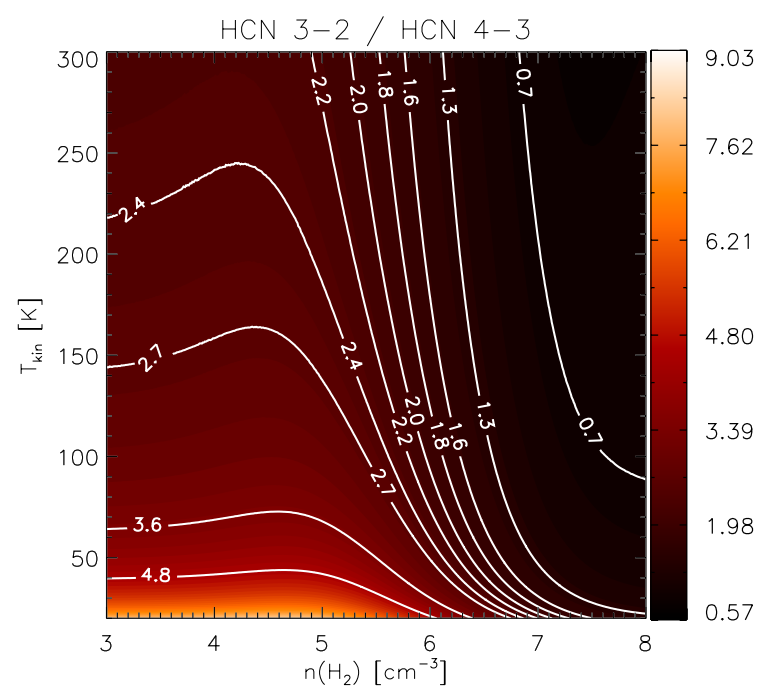

Fig. A.2. Line ratios of $\mathrm{HCN} 3-2$ and $4-3$ as a function of kinetic temperature and $\mathrm{H}_{2}$ density for $F W H M=18 \mathrm{~km} \mathrm{~s}^{-1}$.

\section{Appendix A: Radex line ratio plots}

In Sects. 3.3 and 3.4 we compare the observed line ratios to line ratios calculated using the non-LTE code Radex (Van der Tak et al. 2007). Figure A.1 shows the the $\mathrm{o}-\mathrm{H}_{2} \mathrm{CO} 5_{15}-4_{14}$ and $5_{33}-4_{32}$ line intensity ratios for $\mathrm{H}_{2}$ volume densities between $10^{3} \mathrm{~cm}^{-3}$ and $10^{6} \mathrm{~cm}^{-3}$ and temperatures between $20 \mathrm{~K}$ and 300 K. Figure A.2 shows the HCN 3-2 and 4-3 line intensity ratios for $\mathrm{H}_{2}$ column densities between $10^{3} \mathrm{~cm}^{-3}$ and $10^{8} \mathrm{~cm}^{-3}$ and temperatures between $20 \mathrm{~K}$ and $300 \mathrm{~K}$. Both of these calculations use a molecular column density of $10^{14} \mathrm{~cm}^{-2}$ and a background temperature of $2.73 \mathrm{~K}$. We assume a line width of $F W H M=12 \mathrm{~km} \mathrm{~s}^{-1}$ for $\mathrm{H}_{2} \mathrm{CO}$, and a line width of $18 \mathrm{~km} \mathrm{~s}^{-1}$ for $\mathrm{HCN}$ for the central $40^{\prime \prime} \times 40^{\prime \prime}$, in consistence with the observed line widths.

\section{References}

Aloisi, A., Tosi, M., \& Greggio, L. 1999, ApJ, 118, 302

Alves, J., \& Homeier, N. 2003, ApJ, 589, L45

Bayet, E., Gerin, M., Phillips, T. G., \& Contursi, A. 2004, A\&A, 427, 45

Bradford, C. M., Nikola, T., Stacey, G. J., et al. 2003, ApJ, 586, 891

Brogan, C. L., \& Troland, T. H. 2001, ApJ, 550, 799

Buckle, J. V., Hills, R. E., Smith, H., et al. 2009, MNRAS, 399, 1026

Buckley, H. D., \& Ward-Thompson, D. 1996, MNRAS, 281, 294

De Pree, C. G., Mehringer, D. M., \& Goss, W. M. 1997, ApJ, 482, 307

De Pree, C. G., Wilner, D. J., Mercer, A. J., et al. 2004, ApJ, 600, 286

De Vicente, P. Martín-Pintado, J., \& Wilson, T. L. 1997, A\&A, 320, 957

Dumouchel, F., Faure, A., \& Lique, F. 2010, MNRAS, 406, 2488

Ferrière, K., Gillard, W., \& Jean, P. 2007, A\&A, 467, 611

Genzel, R., Downes, D., Moran, J. M., et al. 1978, A\&A, 66, 13

Goldsmith, P. F., \& Langer, W. D. 1999, ApJ, 517, 209

Green, S. 1991, ApJS, 76, 979

Greve, T. R., Papadopoulos, P. P., Gao, Y., \& Radford, S. J. E. 2009, ApJ, 692, 1432

Gwinn, C. R., Moran, J. M., \& Reid, M. J. 1992, ApJ, 393, 149

Homeier, N., \& Alves, J. 2005, A\&A, 430, 481

Hüttemeister, S., Wilson, T. L., Bania, T. M., \& Martín-Pintado, J. 1993, A\&A, 280,255

Jaffe, D. T., Harris, A. I., \& Genzel, R. 1987, ApJ, 316, 231

Klessen, R. S., Spaans, M., \& Jappsen, A.-K. 2007, MNRAS, 374, L29

Loenen, A. F., Spaans, M., Baan, W. A., \& Meijerink, R. 2008, A\&A, 488, 5

Mangum, J. G., \& Wootten, A. 1993, ApJS, 89, 123

Mauersberger, R., Henkel, C., Weiß, A., Peck, A. B., \& Hagiwara, Y. 2003, A\&A, 403, 561

McCauley, P. I., Mangum, J. G., \& Wootten, A. 2011, ApJ, 742, 58

Meijerink, R., \& Spaans, M. 2005, A\&A, 436, 397

Meijerink, R., Spaans, M., \& Israel, F. P. 2007, A\&A, 461, 793 
Mufson, S. L., \& Liszt, H. S. 1977, ApJ, 212, 664

Mundy, L. G., Evans, N. J., II, Snell, R. L., Goldsmith, P. F., \& Bally, J. 1986, ApJ, 306, 670

Mühle, S., Seaquist, E. R., \& Henkel, C. 2007, ApJ, 671, 1579

Naylor, B. J., Bradford, C. M., Aguirre, J. E., et al. 2010, ApJ, 722, 668

Neufeld, D. A., Lepp, S., \& Melnick, G. J. 1995, ApJS, 100, 132

Ott, J., Weiß, A., Henkel, C., \& Walter, F. 2005, ApJ, 629, 767

Papadopoulos, P. P., van der Werf, P. P., Xilouris, E. M., et al. 2012, MNRAS, in press [arXiv: 1109.4176]

Peng, T., Wyrowski, F., van der Tak, F. F. S., Menten, K. M., \& Walmsley, C. M. 2010, A\&A, 520, A84

Plume, R., Jaffe, D. T., Evans, N. J., II, Martin-Pintado, J., \& Gomez-Gonzalez, J. 1997, ApJ, 476, 730

Plume, R., Fuller, G. A., Helmich, F., et al. 2007, PASP, 119, 102

Rigopoulou, D., Kunze, D., Lutz, D., Genzel, R., \& Moorwood, A. F. M. 2002, A\&A, 389, 374

Roberts, H., Van der Tak, F. F. S., Fuller, G. A., Plume, R., \& Bayet, E. 2011, A\&A, 525, 107

Scoville, N. Z., Sargent, A. I., Sanders, D. B., et al. 1986, ApJ, 303, 416

Serabyn, E., Guesten, R., \& Schulz, A. 1993, ApJ, 413, 571

Schöier, F. L., van der Tak, F. F. S., van Dishoeck, E. F., \& Black, J. H. 2005, A\&A, 432, 369

Sievers, A. W., Mezger, P. G., Bordeon, M. A., et al. 1991, A\&A, 251, 231

Smith, N., Whitney, B. A., Conti, P. S., De Pree, C. G., \& Jackson, J. M. 2009, MNRAS, 399, 952
Snell, R. L., Goldsmith, P. F., Erickson, N. R., Mundy, L. G., \& Evans, N. J., II. 1984, ApJ, 276, 625

Solomon, P. M., \& Vanden Bout, P. A. 2005, ARA\&A, 43, 677

Tielens, A. G. G. M. 2005, The Physics and Chemistry of the Interstellar Medium, 1st edn. (Cambridge: Cambridge Univ. Press)

Troscompt, N., Faure, A., Wiesenfeld, L., Ceccarelli, C., \& Valiron, P. 2009, A\&A, 493, 687

Tsujimoto, M., Hosokawa, T., Feigelson, E. D., Getman, K. V., \& Broos, P. S. 2006, ApJ, 653, 409

Turner, B. E. 1991, ApJS, 76, 617

Van der Tak, F. F. S., Van Dishoeck, E. F., \& Caselli, P. 2000, A\&A, 361, 327

Van der Tak, F. F. S., Black, J. H., Schöier, F. L., Jansen, D. J., \& van Dishoeck, E. F. 2007, A\&A, 468, 627

Vastel, C., Spaans, M., Ceccarelli, C., Tielens, A. G. G. M., \& Caux, E. 2001, A\&A, 376, 1064

Ward-Thompson, D., \& Robson, E. I. 1990, MNRAS, 244, 458

Welch, W. J., Dreher, J. W., Jackson, J. M., Terebey, S., \& Vogel, S. N. 1987, Science, 238, 1550

Williams, J. A., Dickel, H. R., \& Auer, L. H. 2004, ApJS, 153, 463

Wilner, D. J., De Pree, C. G., Welch, W. J., \& Goss, W. M. 2001, ApJ, 550, L81 Wilson, T. L., \& Rood, R. 1994, ARA\&A, 32, 191

Wu, J., Evans, N. J., II, Shirley, Y. L., \& Knez, C. 2010, ApJS, 188, 313

Zhu, M., Seaquist, E. R., \& Kuno, N. 2003, ApJ, 588, 243 